\title{
A Stable Approach to Conical Diffraction of Nearly Lossless Metallic Gratings
}

\author{
Wen-Lan Yeh and Yih-Peng Chiou \\ Graduate Institute of Photonics and Optoelectronics, \\ Graduate Institute of Communication Engineering, and \\ Department of Electrical Engineering \\ National Taiwan University \\ Taipei 10617, Taiwan \\ Email: ypchiou@ntu.edu.tw
}

\begin{abstract}
We adopt a multidomain pseudospectral method to analyze lamellar gratings for general conical diffraction from TE and TM diffractions. The validity and usefulness of this approach are demonstrated through normal metallic lossy and highly conductive lossless gratings. The developed method provides excellent numerical stability and convergence over commonly used rigorous couple wave analysis (RCWA) when the nearly lossless metal is involved. In the case, RCWA suffers from instability problem, while our method still performs well.
\end{abstract}

\section{INTRODUCTION}

Over the past few years, the applications of highly conductive gratings have recently brought to light by the phenomenon of SPP-mediated extraordinary transmission. Therefore, there is a need for an accurate, efficient and stable method to investigate the diffraction and electromagnetic field profiles inside a highly conductive grating. Here, we introduce a numerical model to solve the conical diffraction of nearly lossless metallic gratings [1]. This method is based on applying a spectral accuracy at the Chebyshev collocation points to the spatial derivatives in Helmholtz equation, and then dividing the computational domain into nonoverlapping subdomains [2]. Finally the physical boundary conditions at the subdomain interfaces enforce the subdomains to a global system.

\section{FORMULATION}

The geometry with notations is shown in Fig. 1 and we divide the computational domain into nonoverlapping subdomains as sketched in Fig.2. Each subdomain would be continuous and smooth with refractive index profile $n(x, y)$. Since diffraction problem is invariant in the y-direction and the tangential component would be retained, we make the onset for the total field in a single subdomain expressed as follows:

$(\mathbf{E}, \mathbf{H})(x, y, z)=(e, h)(x) e^{i\left(k_{x} x+k_{y} y+\beta z\right)}, k_{x}=k \sin \theta \cos \phi, k_{y}=k \sin \theta \sin \phi$

where $\beta$ is the propagation constant. To satisfy the Helmholtz equation, the eigenvalue equations in terms of the $E_{x}$ and $E_{y}$ can be derived as follows:

$$
\left[\frac{d^{2}}{d x^{2}}+2 i k_{x} \frac{d}{d x}+\left(k^{2}-k_{x}^{2}-k_{y}^{2}\right)\right] e_{x, y}(x)=\beta^{2} e_{x, y}(x)
$$

To avoid Runge phenomenon and achieve uniform accuracy, we adopt the Chebyshev points instead of the classical uniform interpolation, and then Chebyshev grids are used to construct the differential matrices D. It is the derivative operator which achieves so called "spectral accuracy" in a single domain with a homogenous or smoothly varying dielectric constant. Subdomains in the same horizontal plane in Fig. 2 are merged with the help of boundary conditions across the vertical and horizontal interfaces. The multidomain system can be described as

$$
\begin{aligned}
& {\left[\begin{array}{cccc}
\mathbf{B}_{a, x}^{N} & -\mathbf{B}_{b, x}^{N} & \mathbf{B}_{a, y}^{N} & -\mathbf{B}_{b, y}^{N} \\
\tilde{\Theta}_{a, x} & \mathbf{0} & \mathbf{0} & \mathbf{0} \\
\mathbf{0} & \tilde{\Theta}_{b, x} & \mathbf{0} & \mathbf{0} \\
\mathbf{0} & \mathbf{0} & \tilde{\Theta}_{a, y} & \mathbf{0} \\
\mathbf{0} & \mathbf{0} & \mathbf{0} & \tilde{\Theta}_{b, x} \\
\mathbf{B}_{a, x}^{D} & -\mathbf{B}_{b, x}^{D} & \mathbf{B}_{a, y}^{D} & -\mathbf{B}_{b, y}^{D}
\end{array}\right]\left[\begin{array}{l}
\mathbf{e}_{\mathbf{a}, \mathbf{x}} \\
\mathbf{e}_{\mathbf{b}, \mathbf{x}} \\
\mathbf{e}_{\mathbf{a}, \mathbf{y}} \\
\mathbf{e}_{\mathbf{b}, \mathbf{y}}
\end{array}\right]} \\
& =\beta\left[\begin{array}{cccc}
\mathbf{0} & \mathbf{0} & \mathbf{0} & \mathbf{0} \\
\tilde{\mathbf{I}}_{a, x} & \mathbf{0} & \mathbf{0} & \mathbf{0} \\
\mathbf{0} & \tilde{\mathbf{I}}_{b, x} & \mathbf{0} & \mathbf{0} \\
\mathbf{0} & \mathbf{0} & \tilde{\mathbf{I}}_{a, y} & \mathbf{0} \\
\mathbf{0} & \mathbf{0} & \mathbf{0} & \tilde{\mathbf{I}}_{b, y} \\
\mathbf{0} & \mathbf{0} & \mathbf{0} & \mathbf{0}
\end{array}\right]^{2}\left[\begin{array}{l}
\mathbf{e}_{\mathbf{a}, \mathbf{x}} \\
\mathbf{e}_{\mathbf{b}, \mathbf{x}} \\
\mathbf{e}_{\mathbf{a}, \mathbf{y}} \\
\mathbf{e}_{\mathbf{b}, \mathbf{y}}
\end{array}\right]
\end{aligned}
$$

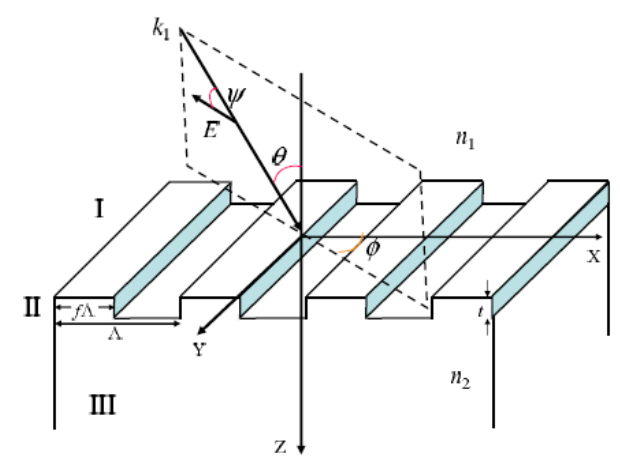

Fig. 1 Schema of a diffraction binary grating in a conical mounting. 


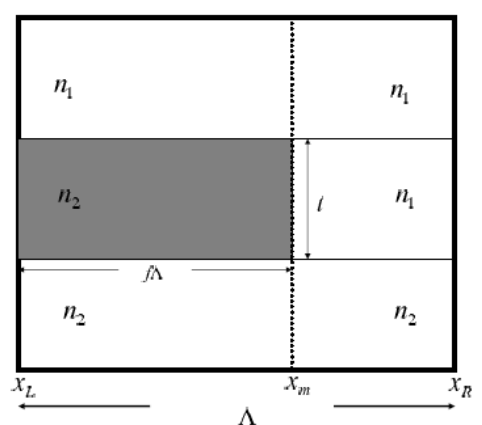

Fig. 2 Cross section of a grating. We divide the computational domain into 6 nonoverlapping subdomains.

where $\boldsymbol{\Theta}$ is the Helmholtz operator in the nonoverlaping subbdomains other than boundary points $x_{m}, x_{R}$ and $x_{L} . B^{D}$ and $B^{N}$ represent Dirichlet boundary condition (DBC) or Neumann boundary condition (NBC), respectively. Solving this eigenvalue problem of (3), we get the propagating constants and mode patterns in such a multidomain system. The total electric field in region $s(s=1,2,3)$ can be expanded in terms of normalized waves corresponding to different modes solved

$$
\begin{gathered}
\mathbf{E}_{s}(x, y, z)=e^{i k_{x} x} e^{i k_{y} y} . \\
\sum_{q=1}^{q=M}\left(e_{s x, q}(x) \hat{x}+e_{s y, q}(x) y+e_{s z, q}(x) \hat{z}\right)\left(v_{s q}^{+} e^{-j \beta z}+v_{s q}^{-} e^{j \beta z}\right)
\end{gathered}
$$

\section{NUMERICAL RESULTS}

The first example is the diffraction of a highly conductive grating with $\Lambda=t=500 \mathrm{~nm}, n=10 i, \theta=30$ degree, and $\lambda=$ $632.8 \mathrm{~nm}$. The numerical instability was pointed out in [3] when the groove width varies from 6 to $460 \mathrm{~nm}$ with $2 \mathrm{~nm}$ intervals. It is an acknowledged criterion for numerical stability with TM polarization for the rigorous couple wave analysis (RCWA) [4]. In Fig. 3, we present a conical case with azimuthal angle $\varphi=45$ degree and the polarization is still kept in $p$-polarization. The red dash line is obtained from commercial tool and the blue line is from our MDPS method. There are many artifacts shown in the dash line because the inversion of the Toeplitz matrices generated by the Fourier coefficients of permittivity distribution. Even we increase orders and the spurious modes would not disappear. In the meanwhile, there is no instability observed in the blue line. This is the first time to our knowledge to simulate the conical diffraction when such highly conductive lossless metal is

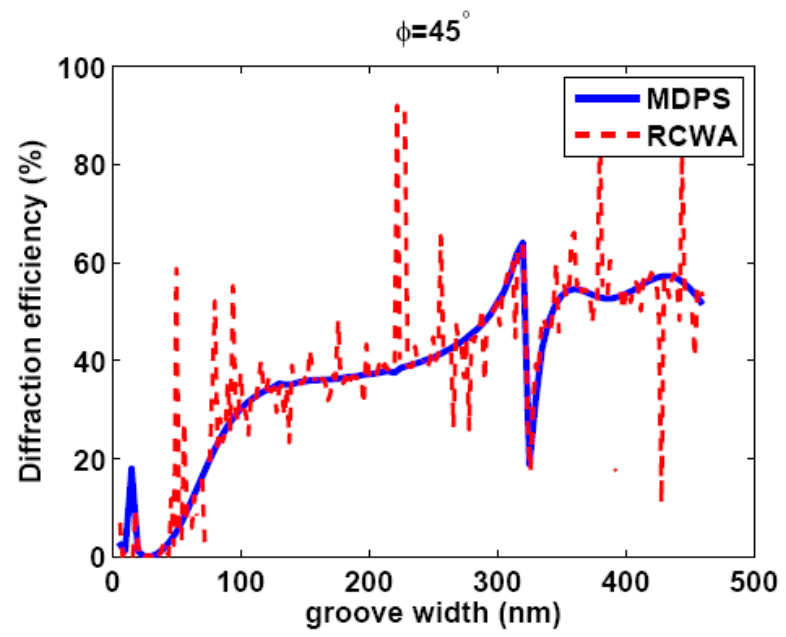

Fig. 3 Diffraction efficiencies as the function of the groove width for a highly conductive lossless grating with $n=0+i 10$ in conical mounting $\varphi=45$ degree.

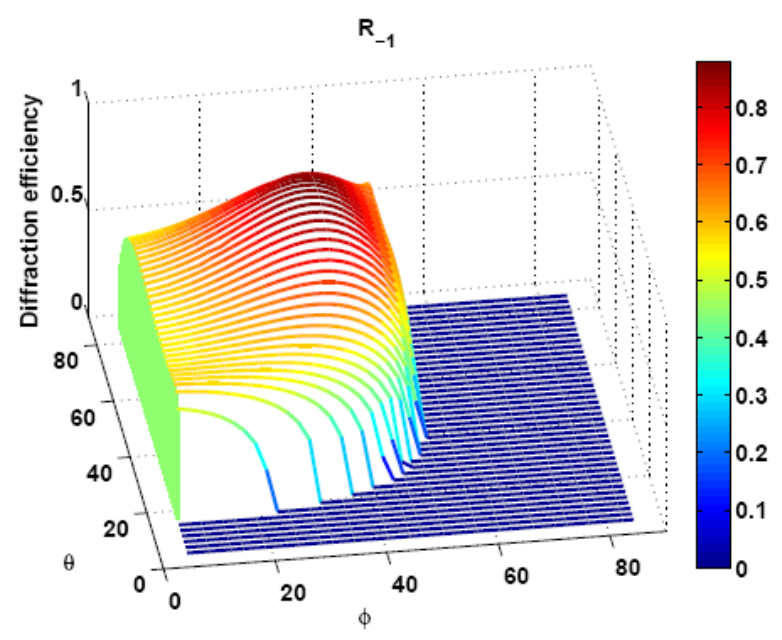

Fig. $4 R_{-1}$ as a function of $\theta$ and $\varphi$.

first minus diffraction efficiency as a function of $\theta$ and $\varphi$. he groove width is $200 \mathrm{~nm}$ and 110 grids are used. Again, there is no instability observed. In addition, the total power of diffraction waves in Fig. 3 and Fig. 4 is equal to the incident wave, which validates our results.

\section{CONCLUSION}

We have developed a powerful multidomain psedudospectral technique to model the conical diffraction of highly conductive lamellar gratings. Simulation results are verified with the widely used RCWA. We not only verified the diffraction efficiencies with the planar diffraction case but also extended to the general conical cases. Both numerical stability and convergence are very good. The new method is stable for gratings with any materials and only reasonable grids are required to get convergent results.

\section{REFERENCES}

[1] Y. P. Chiou, W. L. Yeh, and N. Y. Shih, "Analysis of highly conducting lamellar gratings with multidomain pseudospectral method," IEEE/OSA J. Lightwave Technol., Vol. 27, pp. 5151-5159, 2009.

[2] T. J. Rivlin, The Chebyshev Polynomials, New York: John-Wiley \& Sons, 1974.

[3] E. Popov, B. Chernov, M. Neviere, and N. Bonod, "Differential theory: Application to highly conducting gratings," J. Opt. Soc. Am. A, Vol. 21, pp. 199-206, 2004.

[4] P. Lalanne and G. M. Morris, "Highly improved convergence of the coupled-wave method for TM polarization," J. Opt. Soc. Am. A, Vol. 13, pp. $779-784,1996$. 